\title{
Interventions to Reduce Late Referrals to Nephrologists
}

\author{
Michael J. Fischer ${ }^{a, b}$ Shubhada N. Ahya ${ }^{c}$ Elisa J. Gordon ${ }^{a, d, e}$ \\ ${ }^{a}$ Center for Management of Complex Chronic Care, Edward Hines Jr., VA Hospital, Hines, III., and \\ ${ }^{b}$ Medicine, Jesse Brown VA Medical Center and University of Illinois College of Medicine, \\ ${ }^{\mathrm{c}}$ Medicine, ${ }^{\mathrm{d}}$ Institute for Healthcare Studies, and ${ }^{\mathrm{e}}$ Comprehensive Transplant Center, Feinberg School of \\ Medicine, Northwestern University, Chicago, III., USA
}

\section{Key Words}

Late referral $\cdot$ Chronic kidney disease $\cdot$ Interventions •

Education - Perceptions

\begin{abstract}
Background: Despite the adverse health outcomes with late nephrology referral for patients with chronic kidney disease (CKD), little is known about the underlying mechanisms and reasons for late referral patterns. Methods: We conducted a Medline search for peer-reviewed articles focused on nephrology referral among adults with CKD. We critically reviewed this literature in regard to late nephrology referral, highlighted its shortcomings, and provided a theoretically based framework for future research. Results: Late referral has been attributed to three key types of factors: primary care provider (PCP), patient, and healthcare system factors. Limited empirical research has pointed to knowledge deficits, perceptions, and preferences by PCPs as well as to knowledge deficits, psychological concerns, and economic concerns by patients. The existing literature is severely limited by retrospective designs, closed-ended surveys, and the absence of a theoretically driven conceptual framework to fully evaluate root causes of this ongoing problem. Social Cognitive Theory may be a particularly well-suited concep-
\end{abstract}

tual model for late nephrology referral because important PCP and patient factors correspond to key constructs of this theory. Conclusion: Future research is needed to delineate pathways underlying reasons for PCPs' late referral patterns and CKD patients' referral nonadherence. Interventions are urgently needed to reduce late referrals and improve the health of patients with CKD.

Copyright $\odot 2010$ S. Karger AG, Basel

\section{Introduction}

During the last decade, the prevalent end-stage kidney disease (ESKD) population in the US has continued to increase by more than $20 \%$ and included over half a million individuals by 2008 [1]. ESKD is associated with disproportionately high morbidity, mortality, hospitalizations, and healthcare costs [1]. ESKD patients incur approximately 12 days of inpatient care per year and annual rates of death exceed 150 per 1,000 patient-years [1]. Although ESKD patients comprise less than $1 \%$ of all Medicare beneficiaries, they account for $6 \%$ of Medicare total costs, which were nearly USD 23 billion in 2008 [1].

To optimize treatment for ESKD and prepare patients for dialysis or kidney transplantation, national consensus

\section{KARGER}

Fax +4161306 1234

E-Mail karger@karger.ch

www.karger.com
(C) 2010 S. Karger AG, Basel

0250-8095/11/0331-0060\$38.00/0

Accessible online at:

www.karger.com/ajn
Michael J. Fischer, MD, MSPH

Center for Management of Complex Chronic Care

Hines VA Hospital, 5000 S. 5th Avenue (151H)

Hines, IL 60141 (USA)

Tel. +1 708202 2414, Fax +1 708202 2316, E-Mail fischerm@uic.edu 
guidelines generally recommend that patients with severe chronic kidney disease (CKD) or pre-ESKD [estimated glomerular filtration rate (eGFR) $<30 \mathrm{ml} / \mathrm{min} /$ $1.73 \mathrm{~m}^{2}$ ] be referred to a nephrologist for further evaluation and management [2-4]. It is also suggested that primary care providers (PCPs) should refer their patients to nephrologists when they are unable to complete necessary evaluations of CKD or implement recommended treatments for CKD [4]. Nephrology care for individuals with severe CKD confers considerable health benefits including: treatment of kidney disease complications (e.g., hypertension, anemia, metabolic abnormalities), attenuation of disease progression, informed modality choice and coordinated initiation of renal replacement therapy, timely placement of vascular access if required, and arrangements for kidney transplantation, if appropriate [4, 5]. Multiple studies and meta-analyses have shown that absent, infrequent or late pre-ESKD nephrology care is associated with significantly worse health outcomes, including: substantially prolonged hospitalization and a 2 -fold increase in 1-year mortality [6-9]. Absent and late nephrology referral for ESKD patients has also been demonstrated to negatively impact the quality of pre-ESKD care, resulting in a higher frequency of uremic, metabolic, and anemia complications as well as a lower likelihood of appropriate vascular access, and receipt of peritoneal dialysis or kidney transplantation $[7,8]$. Moreover, late referral is associated with significantly higher costs stemming predominantly from prolonged hospitalization at the time of dialysis initiation, amounting to over an estimated USD 30 million annually [8-11].

Despite the negative consequences of late nephrology referral and the promulgated guidelines regarding its proper indication [2-4], recent reports found that 25$50 \%$ of incident ESKD patients in the US are referred to a nephrologist at a very advanced stage of CKD or not at all $[7,12-17]$. The heterogeneity of nephrology referral rates across studies is partly due to different ways of operationalizing untimely or late referral, which typically is defined as when the index nephrology visit is 1-6 months prior to patients' initiation of dialysis [8]. Regardless of how it is operationalized, untimely or late referral is broadly defined as occurring when pre-ESKD management could have been improved with earlier interactions with a nephrology provider [18].

The factors contributing to late pre-ESKD nephrology care can be categorized into healthcare system-related (e.g., managed care), provider-related (e.g., type of physician), and patient-related (e.g., race, age, disease, insurance) $[7,12,13,15-17]$. Some have attempted to quantify the contribution of these variables toward late nephrology referral, arguing that the majority of late referrals is due to provider and patient factors [12]. Therefore, these two domains are the focus of this review. Little is known regarding the mechanisms underlying the relationships between these identified risk factors and late referral patterns. In the absence of knowledge about these mechanisms, few interventions have been developed and implemented to remedy the problem. In the following discussion, we identify the gaps in knowledge regarding reasons for ongoing suboptimal patterns of pre-ESKD nephrology care, discuss needed areas for future investigation, and propose interventions that may potentially improve timely nephrology referral.

\section{Methods}

In July 2010, we searched Medline for peer-reviewed studies using the following search terms: chronic kidney disease, endstage kidney disease, dialysis, nephrologist, primary care physician, delayed nephrology referral, late nephrology referral, untimely nephrology referral, and intervention. We included crosssectional, retrospective, and prospective observational studies as well as survey research, clinical trials, and review articles published in the English language. We excluded studies conducted in participants $<18$ years old. We used the 'related articles' link in PubMed and reviewed the references of selected articles for additional studies to maximize capture of all relevant publications. We focused on articles published since 1980 .

\section{Late Nephrology Referral: Healthcare System}

Healthcare system-related factors that may contribute to timely nephrology referral include: type of healthcare system, density of nephrologists within a given geographic area, and geographic distance to nephrologists. In one study, patients in a healthcare maintenance organization (HMO) had a 5-fold increased likelihood of a late nephrology referral compared to patients with Medicare [19]. In our own work focusing on elderly veterans, exclusive users of VA outpatient healthcare services and users of both VA and Medicare-covered services were much less likely to have a late nephrology referral than veterans exclusively using Medicare services alone, although referral patterns were uniformly suboptimal [17]. These findings may be related to differences in the integration of healthcare services, clinical information systems and technology, financial incentives for healthcare providers, and patient co-pays for healthcare services. Patients in areas with few nephrologists or a large number of non- 
nephrologists were found to have significantly less likelihood of receiving pre-ESKD care [15]. Studies report inconsistent findings regarding the impact of geography on timely referral: while distance to hemodialysis and healthcare facilities [14, 20] and population density [17] had no impact, pre-ESKD nephrology care was highly variable across hemodialysis centers and geographic regions [16]. Although healthcare system factors can substantially influence patterns of nephrology referral, the fact that late nephrology referral occurs in a variety of countries and healthcare systems $[7,12]$ suggests that provider and patient factors also contribute substantially toward this problem.

\section{Late Nephrology Referral: Providers}

\section{Identified Factors}

The type of primary healthcare provider appears to influence the timeliness of referrals to nephrologists for patients with pre-ESKD [13]. One retrospective US study of incident ESKD Medicare and Medicaid recipients found that nephrology referrals occurred significantly later among patients whose PCP was an internal medicine physician compared with those who had an family medicine physician or other general practitioner [21]. A few European studies have similarly found that late nephrology referrals occur more commonly by internal medicine physicians and other specialists than by general practice physicians $[8,12,22,23]$. The different nephrology referral patterns by physician specialty have been attributed to educational and training disparities, outpatient workload, and customary clinical practice [12]. Other potentially important provider attributes, such as time since completion of postdoctoral training, board certification, medical school, training in kidney disease or referral and practice environment have not been comprehensively evaluated [24].

\section{Potential Mechanisms}

The research on underlying mechanisms for providerrelated late nephrology referral has generally focused on three domains of inquiry: knowledge deficits, perceptions, and preferences.

Knowledge Deficits. Navaneethan et al. [25] surveyed 102 PCPs about their knowledge of CKD and indications for nephrology referral in an upstate New York metropolitan area in 2006. Over 60\% of PCPs were not familiar with the National Kidney Foundation (NKF) Kidney Disease Outcomes Quality Initiative (K/DOQI) clinical practice guidelines (CPGs) and only 31\% indicated that they based nephrology referral on CKD stage as these guidelines recommend. Boulware et al. [26] conducted a national survey study of 178 PCPs (general internists, family physicians, and nephrologists) using hypothetical clinical scenarios of CKD patients. Compared with nephrologists, general internists and family physicians were $40 \%$ less likely to recognize CKD and recommended nephrology referral. As stressed in this article, under-recognition of CKD by PCPs, which in part is related to the failure of testing patients at high risk for CKD, is an important contributing cause of late referral. A subsequent national survey found similar deficits in CKD knowledge among PCPs, particularly among family medicine and older aged PCPs [27]. More recently trained PCPs and those aware of CPGs were substantially more likely to recommend nephrology referral. A recent study of 479 internal medicine residents suggests that nephrology referral decisions among PCPs may be improving as 90\% reported that they would refer a patient with stage $4 \mathrm{CKD}$ or rapidly declining kidney function to a nephrologist [28]. However, poor knowledge of CPGs persists as nearly $1 / 3$ of these physicians in training were not aware of CKD CPGs.

Perceptions. Only three studies have directly inquired into physicians' perceptions about the causes of late nephrology referrals, two of which are over a decade old. In one study involving structured interviews of 86 PCPs between 1982 and 1985 [22], a substantial proportion reported that they perceived negative feedback from nephrologists regarding their referral decisions (43\%), poor communication with nephrologists (37\%), unclear criteria for referral (31\%), and an uncoordinated approach to patient care with the nephrologist during and after referral (23\%) [24]. More recently, Fox et al. [29] conducted in-depth interviews with 10 PCPs and similarly found that PCPs perceived problematic communication with nephrologists, insufficient access to nephrologists, and confusion over coordination of care with nephrologists as contributing factors to poor referral patterns.

In addition to these empirical findings, others have conjectured that late nephrology referrals by PCPs may also be due to their lack of appreciation of benefits of nephrology referral and maintain negative attitudes about nephrology care pre-dialysis. Specifically, PCPs may lack an appreciation of the utility of nephrology care or consultation in the early stages of renal insufficiency $[12,30]$, importance of adequate pre-ESKD care [8], benefits of early referral $[18,36]$, benefits of nephrology care $[18,36]$, severity of the disease [8], meaning of serum creatinine 
concentrations in relation to renal function [30], and value of dialysis for high risk cases, i.e. older, diabetic patients $[5,18,30]$. Regarding negative attitudes, PCPs may view nephrologists as only providing dialysis [18] or merely dealing with technicalities of dialysis [12]. Additionally, PCPs may perceive that they are as adept at managing CKD as nephrologists [12].

Preferences. In a mailed survey study of 728 family practice physicians and general internists in Canada that used hypothetical clinical scenarios focusing on nephrology referral, a preference toward non-referral of patients with older age and comorbid conditions was found [31]. Most providers (67\%) agreed that dialysis rationing was occurring, and that possible criteria for dialysis rationing included the wishes of a competent patient (94\%), short life expectancy (88\%), poor quality oflife (87\%), and age (64\%). While some of these conclusions may be particular to the Canadian healthcare system, similar findings have been noted by studies in the US. Navaneethan and colleagues [25] found that advanced patient age ( $>75$ years), multiple comorbidities and limited life expectancy, and perceived patient non-compliance were reported by PCPs to reduce the likelihood of nephrology referral. These findings suggest that PCPs' subjective judgments about patients' characteristics are influential in referral decisions and thus may be intervened upon to reduce late referrals. Providers may also prefer to not refer patients with severe CKD to nephrologists because of economic issues. These financial concerns include fear of losing clinical responsibility for the patient $[7,8,12]$ and losing income from patients' prescribed clinical care $[7,19]$. In managed care settings, providers may have additional concerns of penalties related to referral of patients for potentially expensive subspecialty care [23]. In our own recent work, where we found a higher rate of timely nephrology referrals for veterans using the Department of Veterans Affairs (VA) for healthcare services compared with those who were not, we speculated that financial incentives to curtail access to nephrology care are less likely to exist within the VA as PCP compensation is unaffected by subspecialty referral, in contrast to Medicare's fee for service environment [17].

\section{Late Nephrology Referral: Patients}

\section{Identified Factors}

Frequently described patient factors associated with nephrology referral patterns are age, race/ethnicity, socioeconomic status, and comorbid chronic conditions.
Although not a unanimous finding $[19,32]$, older age ( $>75$ years) is generally associated with an increased likelihood of late nephrology referral [17, 21, 25, 33-35]. While most studies report that non-white patients (e.g. African-Americans, Hispanics, others) have an increased risk of delayed pre-ESRD nephrology care $[21,25,33,36-$ $38]$, others have not observed this relationship $[17,19,32]$. Less is known regarding the influence of patient's socioeconomic status upon nephrology referral, but a few studies report that lack of health insurance and unemployment are negatively related to timely nephrology referral $[36,37,39]$. The presence of comorbid illness is inconsistently related to timeliness of nephrology referral, ranging from no effect $[17,19]$, to later referrals $[32,37]$, and earlier referrals $[20,21]$. Other less frequently evaluated patient factors, such as gender, have less clear associations with referral patterns [21, 25]. Many have also speculated, but not confirmed through empirical investigation, that a rapid progression of a patient's underlying CKD naturally diminishes a physician's opportunity to provide timely nephrology referral [13]. Compounding this potential concern is the fact that CKD is generally asymptomatic until late in the course of disease progression.

\section{Potential Mechanisms}

Research on underlying mechanisms of patient-related factors contributing to late nephrology referral has focused on four areas: patients' knowledge deficits, negative attitudes, psychological concerns, and economic concerns. No studies have examined patients' attitudes or treatment decision-making about accepting pre-ESKD care, and (non)adherence with nephrology visits and surgery visits.

Knowledge Deficits. Although the association between knowledge deficits and late referral has not been investigated, it has been well documented that many patients have limited awareness of CKD [40], and lack an understanding of the dialysis process $[5,11,12]$. In a recent questionnaire study of 299 US patients with stage 3-4 CKD, an unusually high proportion (89\%) were aware of their CKD diagnosis; however, one-third (31.5\%) of these patients did not know their serum creatinine level [44]. By contrast, an Australian cross-sectional interview study of the public's $(n=852)$ awareness of kidney disease found that participants with CKD had a comparable level of awareness of CKD risk factors (e.g. hypertension, diabetes) as those without CKD. A third of respondents with CKD reported having been tested for kidney function within the past 2 years, and another third indicated 
they had never been tested. Only half (54\%) of those with diabetes and a third (32\%) of those with treated hypertension reported that their kidney function had been tested within the past 2 years [41].

Negative Attitudes. Patients have been found to hold negative attitudes and misconceptions about dialysis, which are believed to contribute to referral nonadherence [11]. CKD patients have been purported to experience psychological concerns including fear of the unknown especially among those who have not observed dialysis $[11,12]$, denial of the progressing disease state [12], refusal to accept the need for dialysis [12], and resistance to begin dialysis until symptomatic [11]. A qualitative study of Colombian patients' coping with CKD revealed that knowledge of the perceived inevitability of dialysis or transplantation caused them great fear which mobilized some to adhere to treatment [42]. A recent systematic review of qualitative studies of treatment decision-making for CKD found that factors influencing patients' treatment choices included having to address one's own mortality, a lack of treatment choice given limited information, the process of obtaining knowledge of options, and the process of weighing alternatives [43].

Economic Concerns. The cost of care has also been identified as a factor driving patients' access to dialysis $[42,43]$; however, it is unknown if costs affect patients' decisions to follow through on seeking subspecialty care like nephrology visits. Nephrology visits among veterans with a service-connected condition or whose income was less than a VA-established annual threshold are free within the VA, while such care necessitates out-of-pocket costs under Medicare. This lesser financial barrier may also contribute to the recently observed more timely receipt of predialysis nephrology care within VA [17]. The extent to which patients' knowledge deficits, attitudes, and economic concerns contribute to late referrals remains to be determined.

\section{Limitations of Existing Literature}

Despite their substantial and known roles in contributing to late nephrology referral, surprisingly little research has investigated PCPs' and patients' perspectives about the barriers to such care. Moreover, although it has been speculated that nephrologists may be contributing to late referral by discouraging PCPs from referring patients with earlier stages of CKD, no research has specifically examined the perspectives of nephrologists regarding timely referral $[22,27,44]$. Specifically, the pro- vider literature is characterized by retrospective studies of clinical practice data, closed-ended surveys of PCPs, and the absence of theoretically driven models that explain the occurrence of late referral. Additionally, few studies assess PCP perspectives in depth or ascertain the association between the prevalence of PCP knowledge deficits or negative attitudes and late referral rates [24,29, 45]. Regarding the patients' perspective, little is known about correlates of patients' nonadherence to nephrology referrals once such referrals have been requested by a PCP. Further, studies have not investigated patients' knowledge, attitudes, perceptions, and beliefs about dialysis or ESKD that are associated with referral nonadherence. Health beliefs are important to be examined as an explanatory factor for treatment adherence [46], and have been shown to contribute to nonadherence to antirejection medications among kidney transplant recipients [47]. Other patient factors such as health literacy, cognitive function, and social support [39] may contribute to late referral, as they do for other barriers in access to care. Qualitative research is needed to reveal the patients' perspective in terms of how they conceptualize barriers to late referral, and the basis of their fear and beliefs about inevitability of dialysis. No theoretical model has as yet been proposed to explain the prevalence or contributing factors of late referral.

\section{Dearth of Interventions}

Only three interventions have been conducted to reduce late referral and/or increase early referral besides the NKF's and VA's global educational initiatives that aim to promote PCPs' use of clinical practice guidelines [3, 4 , 48]. All three of these interventions focused on providers $[45,49,50]$ with only one having also targeted patients [45]. Only one study of these studies was conducted in the US. This US intervention, delivered through Kaiser Permanente, effectively reduced the rate of late referrals by leveraging its integrated electronic medical record (EMR). The intervention granted nephrologists access to the EMR to review all CKD patient records. The EMR stratified patients by level of risk for ESKD onset and prompted nephrologists to initiate consultations to generalists via secure messaging [49]. To ensure intervention sustainability, the number of additional high risk referrals was monitored; only patients with the highest risk profiles were referred if the nephrology group's current workload was maximized, and low risk patients were sent back to primary care, which reduced the rate of unneces- 
sary referrals. A UK study found that electronic consultation using networked EMR supported the PCP's co-management of moderate CKD patients with nephrologists and reduced the need for initial assessment in a hospital clinic [50]. A German retrospective study found that timely referral by PCPs to nephrologists significantly increased over two years after educating PCPs about the health benefits of timely referral and treatment of CKD. Additionally, the intervention involved allaying PCPs' fears that they would reap the economic burden of prescribing costly medications based on recommendations from nephrologists by having nephrologists provide drug prescriptions directly to patients [45].

In recent years, automated eGFR reporting has become widely implemented by clinical laboratories, and appears to increase the rate of CKD recognition and nephrology referrals for patients with CKD outside the US [51-54]. However, the impact of automated reporting on the quality of referrals and health outcomes is unclear. One study found that while the number of nephrology referrals increased after eGFR reporting, the appropriateness of such referrals actually decreased [52]. For example, nephrology referrals of very elderly individuals with mild reductions in kidney function as determined by eGFR reporting (e.g. $50-60 \mathrm{ml} / \mathrm{min} / 1.73 \mathrm{~m}^{2}$ ) without other kidney abnormalities is likely inappropriately early. Hence, strict eGFR-based referrals without appropriate disease context and understanding by providers are generally unwarranted. Thus, adequate provider education regarding CKD is critical. Two studies found that despite eGFR reporting, care of patients with CKD (e.g. use of ACE, ARBs, blood pressure control) had not significantly improved $[51,55]$. As these studies illustrate, eGFR reporting alone without educational interventions appears to be insufficient in changing provider practice behavior $[55,56]$. Furthermore, rates of predialysis nephrology care in recent US studies do not appear to have changed, as up to one-third of patients with severe CKD (eGFR $<30$ $\mathrm{ml} / \mathrm{min} / 1.73 \mathrm{~m}^{2}$ ) still do not receive a nephrology referral and a similar proportion of incident ESKD patients never received pre-ESKD nephrology care $[16,57]$.

With regard to interventions for patients, Canadian multicenter randomized controlled trials of predialysis psychoeducational interventions were found to extend time to dialysis therapy by significant durations relative to usual care $[45,50,58]$ and to increase survival of recipients by a median of 8.0 months longer than usual care after the initiation of dialysis therapy [58]. The psychoeducational interventions entailed educating patients about the normal functions of the kidneys, diseases of the kidneys, nutrition, medications, alternative modes of renal replacement therapy, and lifestyle, while also socializing patients into a collaborative role with service providers to foster increased involvement in self-care [58]. A US structured educational session consisting of providing general information about $\mathrm{CKD}$, and one-on-one instruction from a registered nurse, a dietitian, and a social worker, increased the proportion of patients who received early permanent vascular access placement for dialysis and fistulas rather than grafts or temporary catheters [59]. However, the mechanisms contributing to these relationships remain unknown.

\section{Future Research and Recommendations for Intervention Research}

The development, implementation, and evaluation of practical strategies to improve patterns of nephrology referral for pre-ESKD patients are long overdue, especially considering that the rate of late referral has continued to rise in recent decades $[8,60]$. Many have called for broad educational strategies for both PCPs and patients $[4,6,30]$. While this component is essential for any intervention to facilitate timely referral, too little is known about PCPs' and patients' perceptions, preferences, and beliefs as well as the underlying pathways driving PCP and patient nonadherence to guide a complete and effective intervention. In the following, we present recommendations for future research and interventions to facilitate PCPs' timely referral of CKD patients to nephrologists and ultimately help promote optimal health outcomes.

Foremost, future research is urgently needed to qualitatively assess PCPs', nephrologists', and patients' perceptions about the barriers to and facilitators of timely nephrology referral and referral adherence. For example, although a few qualitative studies have previously identified that PCPs and patients maintain negative beliefs about the value of dialysis, additional qualitative research is essential for describing in depth the meanings associated with dialysis and other disease-related concerns such as CKD progression and cardiovascular events from the PCPs' and/or patients' points of view. PCPs' and patients' complex set of meanings regarding dialysis, renal disease, and its management can be accessed through inquiry into their attributions of the causes of late referral, the values accorded to timely nephrology care, and the effects of late referral, as well as their perceived locus of control or responsibility and self-efficacy for timely refer- 
Fig. 1. Conceptual model of factors influencing late referral.

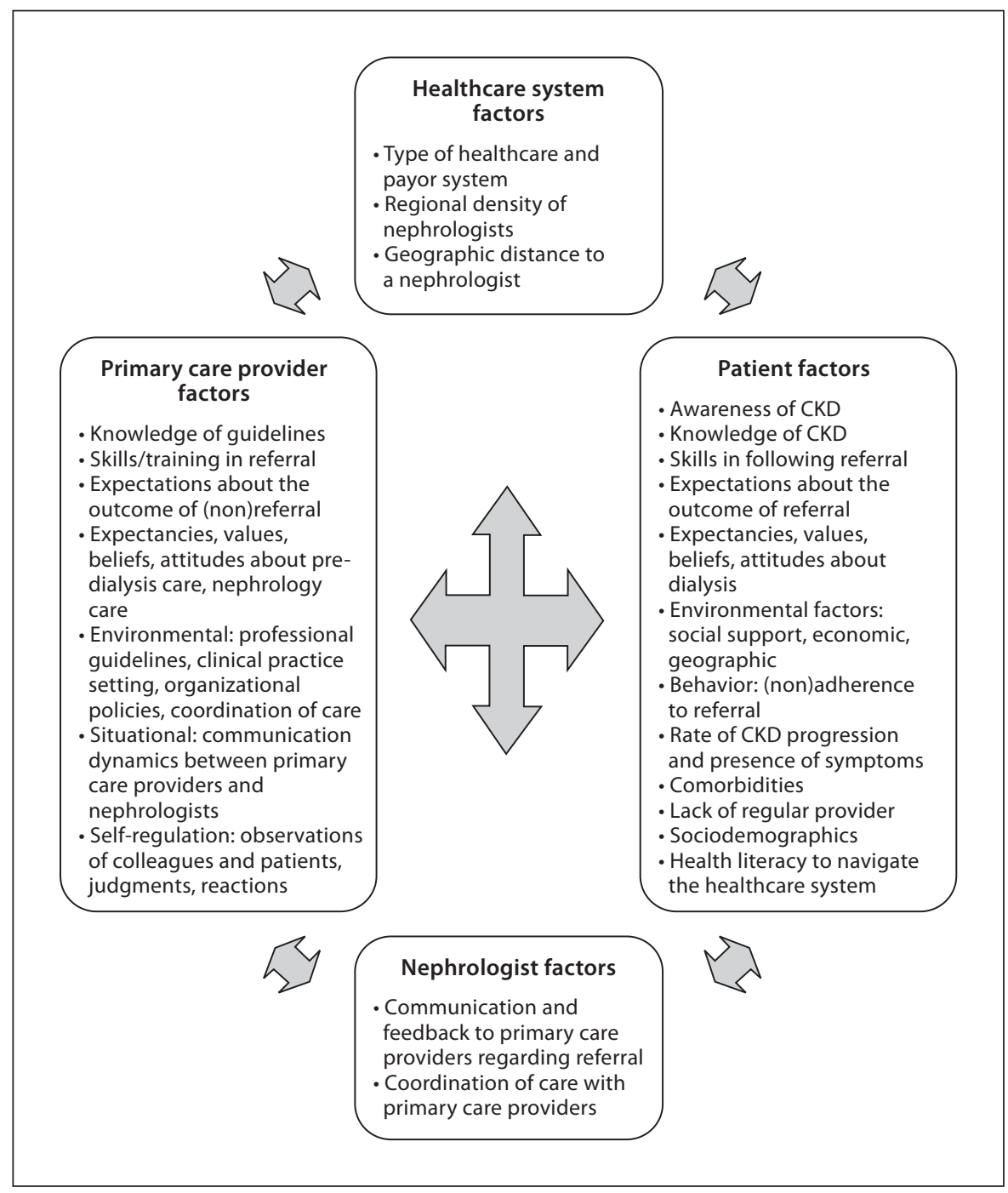

ral or adherence. Identifying the meanings that PCPs and patients ascribe to pre-ESKD care would be valuable for understanding the tacit assumptions that guide their behaviors. Research should also elicit PCPs' and patients' suggestions for ways to remedy the situation, and thereafter assess their receptivity to suggested interventions. Such inquiries are essential to inform effective intervention designs and modes of implementation.

Promising interventions that should be considered include: targeted educational tools for PCPs, improved communication and coordination of care between nephrologists and PCPs, increased collaboration for patient co-management between nephrologists and PCPs, enhancement of resources for PCPs to evaluate complex chronically ill patients, and modification of incentives for subspecialty referral $[26,40,55,61,62]$. A number of future research efforts may be helpful in improving rates of nephrology referral across healthcare systems including: developing methods for identifying patients who will benefit the most from nephrology care, supporting PCP referral decisions and coordination of care with nephrologists [44], and assessing novel ways of healthcare delivery (e.g. multidisciplinary care clinics $[63,64])$ for patients at greatest risk of ESKD [40, 61, 65].

Moreover, educational interventions for CKD patients are needed to ensure comprehension about other outcomes of CKD and the chances of progressing to ESRD. However, further research is needed first to discern patients' information needs and health beliefs associated with pre-ESKD nephrology care. Nonetheless, one de- 
Table 1. Application of social cognitive theory constructs to suggested research on late referral ${ }^{1}$

\begin{tabular}{|c|c|}
\hline Concept & Application to late referral \\
\hline \multicolumn{2}{|c|}{ Personal and behavioral factors } \\
\hline Personal/ & Knowledge of referral guidelines \\
\hline Behavioral & Skills to refer on a timely basis \\
\hline capacity & Attitudes about referral \\
\hline $\begin{array}{l}\text { Expecta- } \\
\text { tions }\end{array}$ & $\begin{array}{l}\text { Beliefs about the anticipated outcomes of timely } \\
\text { and late referral }\end{array}$ \\
\hline \multirow[t]{3}{*}{ Expectancies } & Values placed on timely referral \\
\hline & Attitudes \\
\hline & Beliefs about referral \\
\hline $\begin{array}{l}\text { Self- } \\
\text { efficacy }\end{array}$ & $\begin{array}{l}\text { Confidence in the ability to practice timely } \\
\text { referral and in the ability to overcome barriers to } \\
\text { timely referral }\end{array}$ \\
\hline \multirow[t]{7}{*}{$\begin{array}{l}\text { Self- } \\
\text { regulation }\end{array}$} & $\begin{array}{l}\text { Observations of the impact of referral practices on } \\
\text { patients }\end{array}$ \\
\hline & Response of nephrologists to referral \\
\hline & Colleagues' referral practices \\
\hline & $\begin{array}{l}\text { Judgments: did patient get appropriate } \\
\text { nephrology care? }\end{array}$ \\
\hline & Could timing of referral practice have improved? \\
\hline & Were interactions with nephrologists appropriate? \\
\hline & $\begin{array}{l}\text { Reactions: continue practice or refer patients } \\
\text { earlier }\end{array}$ \\
\hline \multirow[t]{2}{*}{$\begin{array}{l}\text { Positive } \\
\text { reinforce- } \\
\text { ment }\end{array}$} & $\begin{array}{l}\text { From nephrologists, patient, colleagues, clinical } \\
\text { practice report cards } \\
\text { Positive feedback promotes timely referral }\end{array}$ \\
\hline & \\
\hline
\end{tabular}

\section{Environmental factors}

Environ- $\quad$ Factors external to the individual that can affect mental behavior: resources, models, supports

resources Physical environment: clinical practice setting, work flow

Social environment: colleagues, peers

Policy environment: availability of policies, guidelines at local and national levels Coordination of care with nephrologists

Situation

Perceptions of the environment and role within it

Interactions with nephrologists

${ }^{1}$ Adapted from Baranowski et al. [67].

scriptive study that identified the informational needs of patients with stage $4 \mathrm{CKD}$ and their preferences for educational programs found that patients desired knowledge and educational programs about kidney diseases, treatment options, taking prescribed medication, care of a vascular access, and financial concerns [66].
Interventions must be theoretically informed and based on a conceptual model (fig. 1). Social Cognitive Theory (SCT) $[67,68]$ explains health behavior in terms of the reciprocal dynamic between personal, behavioral, and environmental factors (table 1). Thus, unlike many other theories of health behavior, which focus on individuals without consideration of the broader context in which they live, SCT is well suited to guide understanding of late referral because it takes into account how providers' and patients' interactions with their environments influence their knowledge and skills to engage in health behaviors that ultimately affect patients' health outcomes. For example, personal-behavioral factors that are anticipated to affect PCPs' referral practices include knowledge and skills to perform timely referral, expectations or beliefs and attitudes about the outcomes of timely referral, and expectancies or values placed on timely referral. PCPs' self-efficacy, or confidence in their ability to refer patients on a timely basis, is also anticipated to affect their referral practices [68]. Judgments of feeling efficacious in performing timely referral are expected to lead PCPs to carry out the behavior again $[69,70]$. The construct of the environment is especially salient to the issue of late referral given the availability of CPGs and the necessity to interact with nephrologists to coordinate care for CKD patients. Environmental resources are those physically external to the individual (e.g. professional guidelines, organizational policies, interactions with colleagues, and co-management practices), which can provide resources, models, and support for engaging in timely referral. Additionally, the situation or PCPs' (mis)perception of the environment (e.g. interactions with nephrologists) can promote or limit optimal referral practice. SCT is also based on the concept of self-regulation, whereby individuals learn behavior through the processes of observations, judgments, and reactions [68]. That is, PCPs may observe their own or other's (colleagues) referral practices, judge whether the behavior was successful in achieving the goal of helping patients obtain predialysis care, and react to the experience by modifying future referral practices $[69,71]$. Positive reinforcement of timely referral practice (e.g. via positive feedback from nephrologists or patients) may help future timely referral $[69,71]$.

Much remains to be done in order to improve the health of patients with severe CKD and facilitate their transition to and preparation for ESKD therapy. While prior research studies have been informative in delineating provider and patient characteristics associated with nephrology referral patterns, they neither provide insight 
into reasons for these associations nor elucidate causal pathways. Future research is needed to delineate pathways underlying PCPs' late referral and CKD patients' referral nonadherence. Additionally, demonstrating the role of other correlates of late referral, such as the possibility of a lack of usual provider or lack of continuity of care, may prove promising. Moreover, interventions are urgently needed to reduce late referrals and improve the health of patients with severe CKD. By adopting the proposed strategies and a sound conceptual framework, there is great potential for substantially improving the health outcomes for individuals with CKD.

\section{Acknowledgement}

Support was provided by the Department of Veterans Affairs, Veterans Health Administration, Health Services Research and Development Service (VA HSR\&D Career Development Award M.J.F.).

\section{Disclosure Statement}

None of the authors has any conflicts of interest to declare with this paper.

\section{References}

1 US Renal Data System, USRDS: Annual Data Report: Atlas of Chronic Kidney Disease and End-Stage Renal Disease in the United States. Bethesda, National Institutes of Health, National Institute of Diabetes and Digestive and Kidney Diseases, 2009.

$\checkmark 2$ National Institutes of Health: NIH Consensus Statement: morbidity and mortality of dialysis. Ann Intern Med 1994;121:62-70.

3 US Department of Veterans Affairs, Office of Quality and Performance: Clinical Practice Guidelines. http://vaww.oqp.med.va. gov/whatsnew/sitemapdb.asp. Accessed May 6, 2009.

-4 National Kidney Foundation: K/DOQI clinical practice guidelines for chronic kidney disease: evaluation, classification, and stratification. Am J Kidney Dis 2002;39:S1-S266.

5 Huisman RM: The deadly risk of late referral. Nephrol Dial Transplant 2004;19:21752180.

-6 Chan MR, Dall AT, Fletcher KE, Lu N, Trivedi H: Outcomes in patients with chronic kidney disease referred late to nephrologists: a meta-analysis. Am J Med 2007;120:1063.e21070.e2.

7 Obrador GT, Pereira BJ: Early referral to the nephrologist and timely initiation of renal replacement therapy: a paradigm shift in the management of patients with chronic renal failure. Am J Kidney Dis 1998;31:398-417.

$\checkmark 8$ Lameire N, Wauters J, Teruel J, Van Biesen W, Vanholder R: An update on the referral pattern of patients with end-stage renal disease. Kidney Int Suppl 2002;80:27-34.

-9 Jungers P: Late referral: loss of chance for the patient, loss of money for society. Nephrol Dial Transplant 2002;17:371-375.

10 Mendelssohn D, Malmberg C, Hamandi B: An integrated review of 'unplanned' dialysis initiation: reframing the terminology to 'suboptimal' initiation. BMC Nephrology 2009;10.
11 Ismail N, Neyra R, Hakim R: The medical and economical advantages of early referral of chronic renal failure patients to renal specialists. Nephrol Dial Transplant 1998;13: 246-250.

12 Wauters J-P, Lameire N, Davison A, Ritz E: Why patients with progressing kidney disease are referred late to the nephrologist: on causes and proposals for improvement. Nephrol Dial Transplant 2005;20:490-496.

13 Navaneethan S, Aloudat S, Singh S: A systematic review of patient and health system characteristics associated with late referral in chronic kidney disease. BMC Nephrology 2008;9:3.

14 Bradbury BD, Fissell RB, Albert JM, et al: Predictors of early mortality among incident US hemodialysis patients in the Dialysis Outcomes and Practice Patterns Study (DOPPS) Clin J Am Soc Nephrol 2007;2:89-99.

$\checkmark 15$ Zhao Y, Brooks JM, Flanigan MJ, Chrischilles EA, Pendergast JF, Hunsicker LG: Physician access and early nephrology care in elderly patients with end-stage renal disease. Kidney Int 2008;74:1596-1602.

- 16 McClellan WM, Wasse H, McClellan AC, Kipp A, Waller LA, Rocco MV: Treatment center and geographic variability in preESRD care associates with increased mortality. J Am Soc Nephrol 2009;20:1078-1085.

17 Fischer M, Stroupe K, Kaufman J, et al: Predialysis nephrology care among older veterans using Department of Veterans Affairs or Medicare-covered services. Am J Manag Care 2010;16:e57-e66.

18 Eadington DW: Delayed referral for dialysis. Nephrol Dial Transplant 1996;11:2124-2126.

19 Arora P, Obrador G, Ruthazer R, et al: Prevalence, predictors, and consequences of late nephrology referral at a tertiary care center. J Am Soc Nephrol 1999;10:1281-1286.

20 Schmidt RJ, Domico JR, Sorkin MI, Hobbs G: Early referral and its impact on emergent first dialyses, health care costs, and outcome. Am J Kidney Dis 1998;32:278-283.
21 Winkelmayer WC, Glynn RJ, Levin R, Owen WF, Avorn J: Determinants of delayed nephrologist referral in patients with chronic kidney disease. Am J Kidney Dis 2001;38: 1178-1184.

22 Wauters J, Bosson J, Forneris G, et al: Patient referral is influenced by dialysis centre structure in the Diamant Alpin Dialysis cohort study. Nephrol Dial Transplant 2004;19: 2341-2346.

23 Lameire N, Van Biesen W: The pattern of referral of patients with end-stage renal disease to the nephrologist - a European survey. Nephrol Dial Transplant 1999;14:16-23.

24 Campbell J, Ewigman B, Hosokawa M, Van Stone J: The timing of referral of patients with end-stage renal disease. Dial Transpl 1989;18:660-687.

25 Navaneethan S, Kandula P, et al: Referral patterns of primary care physicians for chronic kidney disease in general population and geriatric patients. Clin Nephrol 2010;73: 260-267.

26 Boulware L, Troll M, Jaar B, Myers D, Powe $\mathrm{N}$ : Identification and referral of patients with progressive CKD: a national study. Am J Kidney Dis 2006;48:192-204.

27 Israni R, Shea J, Joffe M, Feldman H: Physician characteristics and knowledge of CKD management. Am J Kidney Dis 2009;54:238-247.

28 Agrawal V, Ghosh AK, Barnes MA, McCullough PA: Perception of indications for nephrology referral among internal medicine residents: a national online survey. Clin J Am Soc Nephrol 2009;4:323-328.

29 Fox C, Brooks A, Zayas L, McClellan W, Murray B: Primary care physicians' knowledge and practice patterns in the treatment of chronic kidney disease: an Upstate New York Practice-based Research Network (UNYNET) Study. J Am Board Fam Med 2006;19:54-61.

30 Levin A: Consequences of late referral on patient outcomes. Nephrol Dial Transplant 2000;15:8-13. 
-31 Mendelssohn D, Toh Kua B, Singer P: Referral for dialysis in Ontario. Arch Intern Med 1995; 155:2473-2478.

- 32 Jungers P, Massy ZA, Nguyen-Khoa T, et al: Longer duration of predialysis nephrological care is associated with improved long-term survival of dialysis patients. Nephrol Dial Transplant 2001;16:2357-2364.

- 33 Ifudu O, Dawood M, Homel P, Friedman EA: Excess morbidity in patients starting uremia therapy without prior care by a nephrologist. Am J Kidney Dis 1996;28:841-845.

- 34 Kessler M, Frimat L, Panescu V, Briançon S: Impact of nephrology referral on early and midterm outcomes in ESRD: EPidémiologie de l'Insuffisance REnale chronique terminale en Lorraine (EPIREL): results of a 2year, prospective, community-based study. Am J Kidney Dis 2003;42:474-485.

- 35 Roderick P, Jones C, Drey N, Blakeley S, et al: Late referral for end-stage renal disease: a region-wide survey in the south west of England. Nephrol Dial Transplant 2002;17: 1256-1259.

36 Kazmi WH, Obrador GT, Khan SS, Pereira BJG, Kausz AT: Late nephrology referral and mortality among patients with end-stage renal disease: a propensity score analysis. Nephrol Dial Transplant 2004;19:18081814.

37 Kinchen KS, Sadler J, Fink N, et al: The timing of specialist evaluation in chronic kidney disease and mortality. Ann Intern Med 2002; 137:479-486.

38 Stack AG: Impact of timing of nephrology referral and pre-ESRD care on mortality risk among new ESRD patients in the United States. Am J Kidney Dis 2003;41:310-318.

- 39 Obialo CI, Ofili EO, Quarshie A, Martin PC: Ultralate referral and presentation for renal replacement therapy: socioeconomic implications. Am J Kidney Dis 2005;46:881-886.

40 Plantinga L, Boulware L, Coresh J, et al: Patient awareness of chronic kidney disease: trends and predictors. Arch Intern Med 2008; 168:2268-2275.

41 White S, Polkinghorne K, Cass A, Shaw J, Atkins R, Chadban S: Limited knowledge of kidney disease in a survey of AusDiab study participants. Med J Aust 2008;188:204-208.

42 Delgado C, Delgado F, Betancourt M, Orozco B: A qualitative study of patient's perceptions of a preventive renal programme in Colombia 2008. Chron Illness 2010;0:1-11.

-43 Morton R, Tong A, Howard K, Snelling P, Webster A: The views of patients and carers in treatment decision making for chronic kidney disease: systematic review and thematic synthesis of qualitative studies. BMJ 2010;340:c112.
44 Renal Physicians Association: Improving early identification and treatment of chronic kidney disease. [URL: http://www.renalmd. org/enews-features.aspx? $\mathrm{id}=1655$ \& terms $=$ referral] accessed October 29, 2010.

45 Mondry A, Zhu A, Loh M, Vo T, Hahn K: Active collaboration with primary care providers increases specialist referral in chronic renal disease. BMC Nephrol 2004;5:16.

46 Conrad P: The meaning of medications: another look at compliance. Social Science and Medicine 1985;20:29-37.

47 Siegal B, Greenstein S: Compliance and noncompliance in kidney transplant patients: cues for transplant coordinators. J Transpl Coord 1999;9:104-108.

48 Wasse H, McClellan W: Increasing physician knowledge about the diagnosis and management of CKD: how we can help primary care providers? Am J Kidney Dis 2009;54:187-190.

49 Lee B, Forbes K: The role of specialists in managing the health of populations with chronic illness: the example of chronic kidney disease. BMJ 2009;339:b2395.

50 Stoves J, Connolly J, Cheung C, et al: Electronic consultation as an alternative to hospital referral for patients with chronic kidney disease: a novel application for networked electronic health records to improve the accessibility and efficiency of healthcare. Qual Saf Health Care 2010;19:e54.

- 51 Hemmelgarn B, Zhang J, Manns B, et al: Nephrology visits and health care resource use before and after reporting estimated glomerular filtration rate. JAMA 2010;303:11511158 .

52 Noble E, Johnson D, Gray N, et al: The impact of automated eGFR reporting and education on nephrology service referrals. Nephrol Dial Transplant 2008;23:38453850.

53 Jain A, McLeod I, Huo C, et al: When laboratories report estimated glomerular filtration rates in addition to serum creatinines, nephrology consults increase. Kidney Int 2009;76:318-323.

-54 Accetta N, Gladstone E, DiSogra C, Wright E, Briggs M, Narva A: Prevalence of estimated GFR reporting among US clinical laboratories. Am J Kidney Dis 2008;52:778-787.

55 Wyatt C, Konduri V, Eng J, Rohtagi R: Reporting of estimated GFR in the primary care clinic. Am J Kidney Dis 2007;49:634641.

56 Akbari A, Swedko PJ, Clark HD, et al: Detection of chronic kidney disease with laboratory reporting of estimated glomerular filtration rate and an educational program. Arch Intern Med 2004;164:1788-1792.

57 Rothberg MB, Kehoe ED, Courtemanche $\mathrm{AL}$, et al: Recognition and management of chronic kidney disease in an elderly ambulatory population. J Gen Intern Med 2008;23: $1125-1130$.
58 Devins G, Mendelssohn D, Barré P, Taub K, Binik Y: Predialysis psychoeducational intervention extends survival in CKD: a 20 year follow-up. Am J Kidney Dis 2005;46: 1088-1098.

59 Lindberg J, Husserl F, Ross J, et al: Impact of multidisciplinary, early renal education on vascular access placement. Nephrol News Issues 2005; 19:35-36, 41-43.

60 Jungers P, Joly D, Nguyen-Khoa T, Mothu N, Bassilios N, Grünfeld J: Continued late referral of patients with chronic kidney disease. Causes, consequences, and approaches to improvement (in French). Presse Med 2006; 35:17-22.

61 St. Peter W, Schoolwerth A, McGowan T, McClellan W: Chronic kidney disease: issues and establishing programs and clinics for improved patient outcomes. Am J Kidney Dis 2003;41:903-924.

62 Rastogi A, Linden A, Nissenson A: Disease management in chronic kidney disease. Adv Chron Kidney Dis 2008;15:19-28.

63 Hemmelgarn B, Manns B, Zhang J, et al: Association between multidisciplinary care and survival for elderly patients with chronic kidney disease. J Am Soc Nephrol 2007;18: 993-999.

64 Curtis B, Ravani P, Malberti F, et al: The short- and long-term impact of multi-disciplinary clinics in addition to standard nephrology care on patient outcomes. Nephrol Dial Transplant 2005;20:147-154.

65 Blantz R: Handing out grades for care in chronic kidney disease: nephrologists versus non-nephrologists. Clin J Am Soc Nephrol 2007;2:193-195.

66 Lewis A, Stabler K, Welch J: Perceived informational needs, problems, or concerns among patients with stage 4 chronic kidney disease. Nephrol Nurs J 2010;37:143-148, quiz 9.

67 Baranowski T, Perry C, Parcel G: How Individuals, Environments, and Health Behavior Interact: Social Cognitive Theory; in Glanz K, Lewis FM (eds): Health Behavior and Health Education: Theory, Research, and Practice, ed 3. San Francisco, Jossey-Bass, 2002, pp 165-184.

68 Bandura A: Self-Efficacy: The Exercise of Control. New York, Freeman, 1997.

69 Clark N, Gong M, Kaciroti N: A model of self-regulation for control of chronic disease. Health Educ Behav 2001;28:769-782.

70 Clark N, Dodge J: Exploring self-efficacy as a predictor of disease management. Health Educ Behav 1999;26:72-89.

71 Prohaska T, Lorig K: What do we know about what works? The role of theory in patient education; in Lorig K (ed): Patient Education: A Practical Approach. Thousand Oaks, Sage Publications, 2001, p 21. 